\title{
Integrated optimization model for location and sizing of offshore platforms and location of oil wells
}

\author{
H.W.L. Rodrigues ${ }^{a}$, B.A. Prata ${ }^{\text {b,*, T.O. Bonates }}{ }^{c}$ \\ a Department of Chemical Engineering, Federal University of Ceará, Campus do Pici, 709 Building, Fortaleza, Ceará, Brazil \\ ${ }^{\mathrm{b}}$ Center of Technology, Federal University of Ceará, Campus do Pici, 710 Building, 60455-900 Fortaleza, Ceará, Brazil

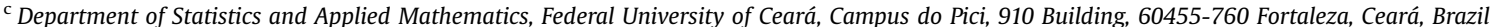

\section{A R T I C L E I N F O}

\section{Article history:}

Received 29 October 2015

Received in revised form

31 March 2016

Accepted 4 July 2016

Available online 4 July 2016

Keywords:

Oil field development

Multicapacitated platforms

Integer programming

Petroleum engineering

\begin{abstract}
A B S T R A C T
The oil field development is a hard and critical task that defines the main procedures to be performed during the oil field productive life. Given the complexity of this planning phase, methods to support decision making have been developed to assist in the proper application of high investments. This paper aims to report a $0-1$ Linear Programming Model which minimizes the development costs of a given oil field as a whole. The model seeks to define: the number, location and capacities of production platforms; number and positions of wells; points where manifolds must be installed; interconnection between platforms, manifolds and wells; and which sections of each well should be vertical or horizontal. The model was named Multicapacitated Platforms and Wells Location Problem (MPWLP). Two different scenarios were tested and the results were consistent with reality, computationally feasible and presented innovations compared to models found in literature.
\end{abstract}

(c) 2016 Elsevier B.V. All rights reserved.

\section{Introduction}

The development of offshore oilfields requires important longterm decisions and they have to be taken in the earliest stage of the project. Many economic, financial, operational and engineering considerations must be taken into account. Nevertheless, at this context, there is the problem of data scarcity and uncertainty about the reservoir and the market, since there is no way to predict with accuracy the actual behavior of the fluids flow or how the oil market will behave for the next few decades.

Concerning the oilfield exploitation, it is necessary to determine, among other parameters, the drainage area; the production method; the number, location, characteristics, and types of wells to be drilled (if producers or injectors; if vertical, directional, horizontal or multilateral); the number and arrangement of the platforms, in the specific case of offshore fields; the operational schedule; the distribution of flowlines, risers and manifolds; the processing plant that should be installed at each platform.

According to the Concession Contract for Exploration, Development and Production of Oil and Natural Gas (Agência Nacional do Petróleo, Gás e Biocombustíveis - ANP, 2013), all these initial decisions should be made in a short period of 180 days after the

\footnotetext{
* Corresponding author

E-mail addresses: hydrawalesca@alu.ufc.br (H.W.L. Rodrigues), baprata@ufc.br (B.A. Prata), tb@ufc.br (T.O. Bonates).
}

declaration of the field commerciality, in the particular case of the Brazilian territory. Apart from their complexity, such decisions affect the behavior of production over time, the recovery factor that can be achieved, the future decisions, the revenue, the economic analysis and, consequently, they have outcomes in all activities during the oilfield's productive life.

Given the problem's multi-criteria nature, the impact of defining the development plan and the great outlays of capital needed in such operations, it is clear that effective decision support methods are required by the designers and managers in this planning phase.

The first relevant work about optimization of offshore oil fields development was published by Devine and Lesso (1972). They presented a general model for developing an oilfield at minimum cost. The decision variables were number, capacity and location of the platforms, and allocation of the drilling targets to each platform. They assumed fixed platforms, directional wells and a continuous space. The mathematical programming model of the problem was shown to be identical, in general structure, to the well-known warehouse location problem and heuristics for solving it were developed. The solution procedure did not determine the number of platforms, so an arbitrary number was considered in each instance. The problem proved to be computationally hard to treat and nowadays it would probably be solved with metaheuristics.

The above-mentioned work was extended by Frair and Devine (1975). They included the time schedules for placing platforms and 
drilling wells and the production schedule for each reservoir. They formulated a Mixed-Integer Non-linear Programming (MINLP) model that aims at maximizing the discounted after-tax cash flows, subject to production limiting constraints. Because of the non-linear nature and size of such model for realistic problems, they divided it into two sub-models: the first one was the location of platforms and allocation of wells and the second one was the schedule for drilling wells. The solution for the first sub-problem was the same that was described in the Devine and Lesso (1972) work.

Grimmett and Startzman (1988) proposed a mathematical programming computational tool, using the branch-and-bound algorithm, in order to model and solve the problem. The objective function was the minimization of the investment, subject to capacity and technology constraints over integer variables.

Hansen et al. (1992) proposed a model of location and sizing of offshore platforms for oil exploration and production as an integer programming multicapacitated plant location problem. The objective function was the minimization of investment costs. They considered directional wells, fixed platforms with predefined sizes and a continuous space. The authors used a general-purpose integer programming model with redundant constraints for medium-size problems. For larger problems, they implemented a Tabu Search heuristic.

Rosa (2006) developed an exhaustive search model with the objective of maximizing the NPV. He linked the concepts of graph theory and pressure gradient in multiphase flow. The author estimated the NPV for all nodes and found the zone of maximum NPV and minimum risers and flowlines costs. In this model, the wellhead positions are known, there are no manifolds or capacity restrictions for the platforms, risers and flowlines (production or injection) costs are the same, the costs of drilling any well or installing a platform is fixed. As the method consists in systematically enumerating all possible candidates, it is clear that the computational time increases dramatically with the number of possibilities for locating a platform. Rosa and Ferreira Filho (2012) extended this work by including the manifolds' locations and the interconnections among them and wells as decision variables.

Evolutionary computational techniques have been used for solving wells placement problems. Bittencourt (1997) presented a hybrid algorithm based on direct methods such as Genetic Algorithm, polytope search method, Tabu Search and memory strategy. His objective function was the maximization of the NPV and consists of a cash flow analysis for production profiles, obtained from simulation runs. Other similar works include Guyaguler and Horne (1999), Yeten (2003), Almeida (2003), Túpac (2005), Emerick et al. (2009), Pacheco and Vellasco (2009), Ebadat and Karimaghaee (2012), Rahmawati et al. (2012) and Tavallali et al. (2013).

The aforementioned works broach different perspectives of the problem of locating wells and platforms, but all of them do this independently and, most of them, from the point of view of the strong combinatorial nature of the problem, bring propositions and intense experimentations of algorithms for solving it in an attempt to reduce the computational time.

The present paper aims at presenting an innovative $0-1$ Linear Programming model for the development of a given offshore oilfield by determining the following factors: the number, location and sizes of the offshore producing platforms; the number and location of wells; the manifolds positions; the interconnection among platforms, manifolds and wells; and which wells sections should be vertical or horizontal. The objective of this model is the minimization of the overall offshore oilfield development cost.

Traditional approaches to locate offshore production platforms usually aim to minimize the investment costs in facilities for the crude oil and gas extraction or maximize the Net Present Value
(NPV) of the achievement. The NPV maximization approach requires production curves for each possible well, while this methodology must be applied to undeveloped oilfields. However, estimating future production in this context involves considerable uncertainty. Even with a great effort to calculate a NPV closer to reality, the production curves are very sensitive to changes in assumptions made during the reservoir modeling as well as changes in the exploitation strategy of the field, which may occur over time. On the other hand, some claim that the investments have greater degree of certainty and the prediction of costs in an undeveloped oilfield can bring consistent results on improving the profitability of the project as a whole. Based on that, we preferred, at first, to use the minimizing investments approach instead of maximizing the NPV.

It can be seen that the problem of locating and sizing production platforms and wells is an extremely complex decision-making process, as it involves several criteria, some of which might conflict with each other. Even subproblems related to wells and platforms location have been approached using simplifications in their general formulation, since the computational time required for their solution is often prohibitive. Modeling this type of problem implicates some tradeoff between realistic representation of the system and solution accuracy versus CPU-time requirements. The MPWLP model proposed here demonstrates a considerable improvement over previous methods of modeling this process, as it integrates interconnected decisions without forgoing an optimal solution. The integer linear programming approach used, with its novel constraints, guarantees an optimal solution within a very reasonable computational time, unlike a MINLP approach, for example, that is able to represent the problem with more accuracy, but oftentimes does not provide a global optimum, due to the nonlinear nature of the modeling, while often consuming excessive CPU time.

The largest fraction of offshore oilfields development costs covers the equipment, pipes of production wells and flowlines that connect these wells to platforms (FRANCO, 2003). Therefore, a good choice of wells and platforms locations allows a significant decrease in the costs, reducing the distances to be traversed by the pipelines and the need for additional production system equipment (Rosa, 2006).

The rest of this paper is structured as follows: in the next section, the problem under study is stated in detail; in the third section, a new formulation for the MPWLP model is presented; in the fourth section, the computational results are shown; lastly, in the final section, we present some conclusions and suggestions for future research.

\section{Problem statement}

An offshore production system consists of one or more Stationary Production Units (SPUs), surface equipment (located on the deck of the platform) and subsurface equipment (located on the seabed, constituting the submarine layout). Technical, environmental and economic aspects may influence the choice of a SPU and, consequently, the choice of the subsea system. The type of platform and the determination and location of equipment depend on factors such as water depth, type of structure used, environmental and weather conditions, flow production system, aspects of the wells (architecture, path, type of completion and arrangement), among others.

All oil extracted from the reservoir through the wells is sent to the SPU through risers and the number of direct connections between the wells and the platform is limited. The control of the quantity of this direct links is performed by manifolds, which are typically located on the submarine ground and act as oil 
concentrators.

The optimization problems related to oil wells and platforms location, in the context of the initial offshore oil field development, are usually treated separately. Nevertheless, it is generally suboptimal to treat the platforms and wells placement optimization problems apart. An integrated model, in turn, simultaneously optimizes decision variables of different but also interdependent functions to perform an overall optimization. Usually, the output of one function can be used as the input of the other (Sarmiento and Nagi, 1999). Thus, an integrated approach to both problems was sought here, with the aim of reducing the overall cost of developing oil fields, since the location of platforms and wells are rather interconnected decisions. In addition, the determination of the production units' capacities to be installed was also taken into consideration, as this decision is strongly related to the total flow of the wells allocated to each of these platforms.

In this approach, we are considering multicapacitated platforms and producer wells with the possibility of having vertical or horizontal stretches. The sea surface, the seabed and the reservoir are represented by three distinct and overlapped graphs, in order to discretize these areas. The vertices of each graph have coordinates $(x, y, z)$ and they represent: the possible locations for platforms (on the sea surface level); the possible locations for manifold and wells (on the seabed level); and the possible locations for targets to be achieved by the wells (on the reservoir level). The representation can become more accurate with the increase of the number of vertices and edges in the graph.

It can be observed that the adoption of the Cartesian coordinate $z$ is of fundamental importance in capturing the topographical peculiarities of the submarine ground and the non-planar properties of most oil reservoirs. Bathymetry data would be ideal for the generation of these graphs, but in the absence of real data, they were only reasonably estimated.

The edges represent the distances between nodes and they were considered here as having the same size. The distances between a possible manifold position in relation to possible wellhead positions are calculated from the Euclidean distance between each pair of vertices and they represent the lengths of flowlines. Thus, the calculation of the flowlines cost was done according to the following equation

$C f l_{i j}=C_{f l / m} \cdot \sqrt{\left(x_{i}^{f}-x_{j}^{f}\right)^{2}+\left(y_{i}^{f}-y_{j}^{f}\right)^{2}+\left(z_{i}^{f}-z_{j}^{f}\right)^{2}}$,

where:

$C f_{i j}$ : cost of the flowline that connects the manifold at site $i$ to the wellhead at site $j$;

$C_{f l / m}$ : unitary cost per meter of flowlines;

$\left(x_{n}^{f}, y_{n}^{f}, z_{n}^{f}\right)$ : Cartesian coordinates $(x, y, z)$ of each possible manifold $(n=i)$ or well $(n=j)$ site $n$ at sea floor, where $n \in\{i, j\}$.

On the other hand, the lengths of the horizontal stretches are calculated using the Manhattan distance between pairs of vertices in the reservoir level, according to Eq. (2).

$\operatorname{Chd}_{j t}=C_{h d / m} \cdot\left(\left|x_{j}^{r}-x_{t}^{r}\right|+\left|y_{j}^{r}-y_{t}^{r}\right|+\left|z_{j}^{r}-z_{t}^{r}\right|\right)$,

where:

Chd $d_{j t}$ : cost of horizontal drilling that connects the end of the vertical well section at site $j$ to the target bottom hole at site $t$; $C_{h d / m}$ : unitary cost per meter of horizontal drilling;

$\left(x_{k}^{r}, y_{k}^{r}, z_{k}^{r}\right)$ : Cartesian coordinates $(x, y, z)$ of each possible end of a vertical well section $(k=j)$ or target $(k=t)$ site $k$ at reservoir, where $k \in\{j, t\}$.

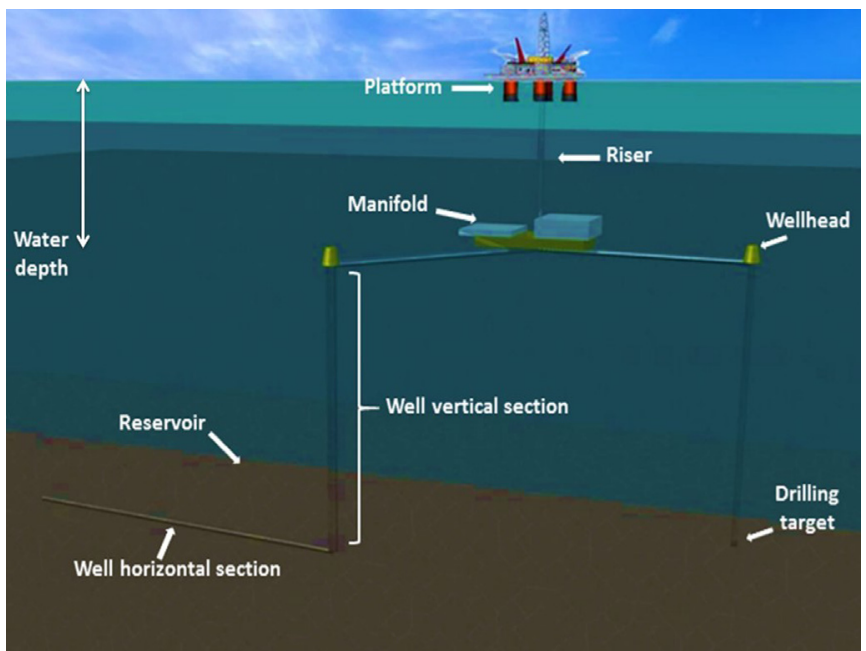

Fig. 1. Schematic drawing of an oil field with the above considerations.

The Manhattan distance consideration is necessary because it restrains the horizontal drilling to the graph edges and, consequently, ensures that wells do not cross each other, an impossible event to happen in real reservoirs. When a reservoir's graph edge is selected to be a section of a horizontal well, one of the restriction equations will ensure that this same segment will not be selected again. If we consider Euclidean distances for the horizontal portions, there would be no simple way to ensure that they do not cross each other.

The fact that the graphs are overlapped results in a simplification: the risers and vertical sections of wells will always be perpendicular to the graphs, because they connect vertices that are immediately below each other. So, the costs of risers and vertical drilling are calculated according to Eqs. (3) and (4) respectively. Fig. 1 illustrates the above considerations used in the model.

$C r_{i}=C_{r / m} \cdot\left(z_{i}^{l}-z_{i}^{f}\right)$,

$C v d_{j}=C_{v d / m} \cdot\left(z_{j}^{f}-z_{j}^{r}\right)$,

where:

$\mathrm{Cr}_{i}$ : cost of the riser that connects the platform at site $i$ to the manifold just below;

$C v d_{j}$ : cost of vertical drilling associated to the well at site $j$;

$C_{r / m}$ : unitary cost per meter of risers;

$C_{v d / m}$ : unitary cost per meter of vertical drilling;

$\left(x_{i}^{l}, y_{i}^{l}, z_{i}^{l}\right)$ : Cartesian coordinates $(x, y, z)$ of each possible platform location $i$ at sea level.

In the proposed model, the distance from the platform to shore is also considered, because it influences directly in both development costs and maintenance and production costs. If the distance from the production unit to the coast is minimal, there will be a reduction in the pipelines installation costs, a lower pressure drop in the fluid flow in these ducts and less will be spent on transportation of goods and personnel. The installation cost of an export pipeline that connects a platform to a receiving facility at shore is calculated as follows:

$C e_{i}=C_{e / m} \cdot\left[\left(z_{i}^{l}-z_{i}^{f}\right)+\sqrt{\left(x^{c}-x_{i}^{l}\right)^{2}+\left(y^{c}-y_{i}^{l}\right)^{2}}+\left(z^{c}-z_{i}^{f}\right)\right]$, 
where:

$C e_{i}$ : cost of the export pipeline that connects the platform at site $i$ to the shore, transporting the oil and gas extracted;

$C_{e / m}$ : unitary cost per meter of export pipelines;

$\left(x^{c}, y^{c}, z^{c}\right)$ : Cartesian coordinates $(x, y, z)$ of the receiving facility at coast where the export pipelines achieve.

In some cases, there may be environmental or safety constraints that could prohibit the installation of manifolds, wells or flowlines in certain points. These underwater obstacles may be considered in the graph representation of the seabed by assigning high values for the adjacent edges of restricted vertices or just by the elimination of these edges.

An estimated oil flow value is associated with each wellhead possible position and the well will produce this oil flow rate if drilled at that point. Such oil flow rates vary over the production time and they can be obtained through reservoir simulation, correlations for pressure gradient in multiphase flow, black oil correlations and reservoir characteristics that vary as a function of time. These values will influence in determining the platforms capacities to be installed. Concerning the platform costs, they vary with the processing capacity needed to receive the entire oil and gas volume produced.

Similarly to the real cases, it is assumed here that only targets in the reservoir to be achieved by the wells are known.

\section{Proposed model}

In this problem, to which we refer as the Multicapacitated Platforms and Wells Location Problem - MPWLP, we intend to obtain the optimal location of platforms, manifolds and wellheads and the interconnection among them, besides the size of platforms, in order to minimize the global cost of the oil field development. Hereafter, the notation used for the formulation of the model will be presented.Indexes and sets

$i \in I \quad$ set of potential locations for platforms;

$j \in J \quad$ set of potential locations for wells;

$t \in T \quad$ set of possible drilling targets;

$s \in S \quad$ set of platform sizes.

Parameters

$C p_{s} \quad$ cost of installing a platform of size $s$

$b_{s} \quad$ maximum processing capacity of a platform of size $s$, generally given in barrels per day (bbl/d).

$q_{j} \quad$ estimated oil production rate at well $j$, generally given in $\mathrm{bbl} / \mathrm{d}$.

$M \quad$ an appropriately chosen large number.

$Q_{\max } \quad$ maximum estimated oil production rate that the entire field can provide, generally given in bbl/d.

\section{Decision variables}

$x_{i j} \quad 0-1$ variable equal to 1 if well $j$ is associated to a platform at site $i$ and 0 otherwise.

$y_{\text {is }} \quad 0-1$ variable equal to 1 if a platform of size $s$ is built at site $i$ and 0 otherwise.

$z_{j t} \quad 0-1$ variable equal to 1 if well $j$ reaches the target at site $t$ and 0 otherwise.

Objective function

$$
\begin{aligned}
\min Z= & \sum_{s \in S} C p_{s} \cdot \sum_{i \in I} y_{i s}+\sum_{i \in I} C r_{i} \cdot \sum_{s \in S} y_{i s}+\sum_{i \in I} \sum_{j \in J} C f l_{i j} \cdot x_{i j}+\sum_{j \in J} C v d_{j} \\
& \cdot \sum_{i \in I} x_{i j}+\sum_{j \in J} \sum_{t \in T} C h d_{j t} \cdot z_{j t}+\sum_{i \in I} C e_{i} \cdot \sum_{s \in S} y_{i s}
\end{aligned}
$$

Constraints

$\sum_{i \in I} x_{i j} \leq 1, \forall j \in J$

$\sum_{s \in S} y_{i s} \leq 1, \forall i \in I$

$\sum_{s \in S} y_{i s}+\sum_{j \in J} x_{j i} \leq 1, \forall i \in I$

$\sum_{i \in I} x_{i j}=\sum_{t \in T} z_{j t}, \forall j \in J$

$M \cdot \sum_{s \in S} y_{i s} \geq \sum_{j \in J} x_{i j}, \forall i \in I$

$\sum_{s \in S} y_{i s} \leq \sum_{j \in J} x_{i j}, \forall i \in I$

$\sum_{j \in J} x_{i j} \cdot q_{j} \leq \sum_{s \in S} b_{s} \cdot y_{i s}, \forall i \in I$

$\sum_{j \in J} x_{i j} \cdot q_{j} \leq Q_{\max }, \forall i \in I$

$\sum_{j \in J} z_{j t}=1, \forall t \in T$

$x_{i j}, y_{i s}, z_{j t} \in\{0,1\}, \forall i \in I, j \in J, s \in S, t \in T$

Objective function (6) minimizes the global cost required in the development plan of a given offshore oil field. The first term refers to the installation cost of the platforms. The second one represents the installation cost of risers connecting platforms and manifolds, while the third term is the total cost of flowlines setting. The fourth term includes the total cost of vertical drilling in all wells. The fifth one represents the costs of horizontal drilling in all wells. Finally, the last term is the cost of installing export pipelines, in order to drain the oil and/or gas produced on each platform.

The set of constraints (7) ensures that each possible well location $j$ can only be connected to, at most, one manifold $i$ (such connection will occur if the well is installed at site $j$ ). The set of constraints (8) establishes that, at each point $i$, there can only be, at most, one platform of size $s$, with capacity $b_{s}$. The constraints of type (9) prohibit any well from being drilled in a point that already has a manifold. The set of constraints (10) ensures that if a well is drilled at location $j$ on the seafloor, the drilling continues vertically until it reaches the corresponding node $j$ in the reservoir level, just

Table 1

Unitary cost values used in the offshore oil field development cost calculation.

\begin{tabular}{ll}
\hline \multicolumn{2}{l}{ Unitary costs (U.S.S/m) } \\
\hline Risers & 1500 \\
Flowlines & 700 \\
Vertical drilling & 3000 \\
Horizontal drilling & 12,000 \\
Export pipelines & 800 \\
\hline
\end{tabular}


Table 2

Input data of case study 1 .

\begin{tabular}{ll}
\hline Case study 1: input data & \\
\hline Graph's dimensions (km) & $15 \times 15$ \\
Edge length (m) & 1000 \\
Water depth range (m) & $1100-1500$ \\
Reservoir depth range (m) & $2000-2700$ \\
Number of targets & 22 \\
Flow rate per well (bbl/d) & $6000-15,000$ \\
Maximum oil field production rate (bbl/d) & 160,000 \\
Coast coordinates X, y, z (m) & $(15,000 ; 300 ; 20)$ \\
Platform cost (U.S.\$) & 800 million \\
Platform capacity (bbl/d) & 200,000 \\
\hline
\end{tabular}

below. Constraints (11a) and (11b) are responsible for the interconnection between the levels of sea surface and seafloor. They establish that if a manifold is installed at site $i$ on the seabed, a platform must be put at the corresponding node $i$ on the sea surface, just above. The set of constraints (12) determines the capacity of the platform $i$ should not be exceeded. Constraints of type (13) ensure that the maximum flow rate that the reservoir is capable of producing will be not exceeded. This last set of restrictions is necessary especially when the data of wells flow rates are randomly generated. Estimated values of wells flow rates that are generated by reservoir simulation already fulfill the set of constraints (13). Constraints of type (14) ensure that all drilling targets have to be achieved and only once. Finally, the set of constraints (15) defines the domain of the model variables.

\section{Solving real-world problems}

\subsection{Case study 1}

Data were taken from an instance problem, which can be found in Rosa (2006). The sea surface, seafloor and reservoir were represented by Cartesian networks with $15 \times 15$ vertices, generating graphs with 225 vertices. The distance between edges that were parallels to the Cartesian plane was $1000 \mathrm{~m}$. Rosa (2006) considered a constant water depth of $1100 \mathrm{~m}$. Here, we assumed a water depth ranging between 1100 and $1500 \mathrm{~m}$. Underwater obstacles were not included in any case, primarily.

The abovementioned author did not provide data about the drilling targets locations, about the distance to the coast or about the unitary costs of drilling (he considered that the cost related to the drilling of any well is fixed). Thus, a reasonable assumption was made here: the positions of the 22 wells of Rosa's problem, provided as input data, are considered here as the drilling targets locations. The author admitted no criteria in the choice of vertices that represent the production and injection wells. Relating to the cost of the platform installation, we used the value set by him, U. S. $\$ 800$ million.

Based on oil production data simulated by Rosa (2006), the oil flow rate values per well were estimated to range between 6000 and $15,000 \mathrm{bbl} / \mathrm{d}$ and the maximum estimated oil production rate

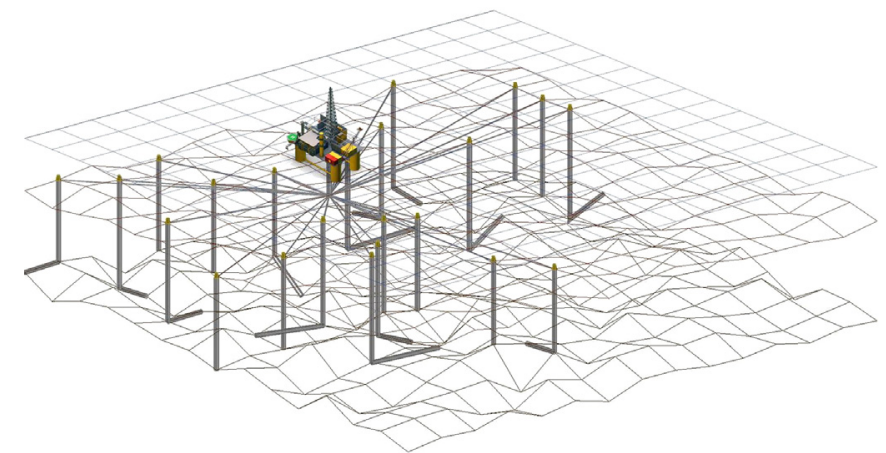

Fig. 2. Oilfield layout obtained in the computational experiment R5 of case study 1.

that the entire field could provide was set as being $160,000 \mathrm{bbl} / \mathrm{d}$. The coordinates of the coast were considered as being $(15,000$; $300 ; 20) \mathrm{m}$. The processing capacity for the possible platform (s) was set as $200,000 \mathrm{bbl} / \mathrm{d}$. Finally, the depth of the reservoir was evaluated between 2000 and $2700 \mathrm{~m}$ below the seafloor. The values of unitary costs used are shown in Table 1 and the input data used on the computational experiments are summarized in Table 2.

Based on the data above, the costs and the coordinates of targets and nodes were generated by a program developed by us in the $C$ language, by implementing Eqs. (1)-(5); they were used in the model as input data in a matrix format. The model Eqs. (6)(15) were implemented and run using IBM CPLEX optimizer software, version 12.5.1. Any Mixed Integer Programming (MIP) solver that can handle large-scale models (with thousands of variables and constraints) could be applied to run our model. CPLEX uses branch-and-cut search, a method of combinatorial optimization for solving Integer Linear Programming (ILP) problems that involves running a branch-and-bound algorithm and using cutting planes to tighten the linear programming relaxations. It is worthnoting that, in MPWLP model, all the decision variables are integer and binary, which means that the model was amenable to solution via MIP techniques, in particular, with the use of a solver equipped with a state-of-the-art branch-and-cut implementation.

Finally, our program automatically generates AutoCAD script files, which are used for plotting the submarine layout obtained from the model's solution. The computational experiments were performed on a computer with Intel Core i7-3770S CPU $3.10 \mathrm{GHz}$ and 8 GB RAM memory.

As can be noticed, data varying within carefully chosen ranges were used (water depth, reservoir depth, flow rate per well), since specific values for each node were not available. In order to estimate the uncertainties involved on generating these oriented random amounts, we tested five distinct instances with the same input data presented in this section. Each time, the model was run, new values within those ranges were obtained, while the others remained the same.

The model results are given on Table 3 . In addition to the instance name (column 1), the table shows, respectively, the following parameters of the model: the optimum solution value for objective function; the percentage reduction on the total

Table 3

Results obtained for five different instances for case study 1 .

\begin{tabular}{|c|c|c|c|c|c|c|c|c|c|c|}
\hline Inst & Cost (U.S.S) & Dif. \% & Plat. position & Horiz. wells & $Q_{t}(b b l / d)$ & Nodes & Const. & Iter. & Var. & Time (min) \\
\hline $\mathrm{R} 1$ & $1,279,421,832$ & -23.8 & 96 & 16 & 159,916 & 16,836 & 2025 & 583,584 & 101,476 & 5.08 \\
\hline $\mathrm{R} 2$ & $1,283,293,457$ & -23.6 & 96 & 16 & 159,907 & 17,765 & 2025 & 509,644 & 101,476 & 4.91 \\
\hline R3 & $1,248,058,012$ & -25.7 & 81 & 15 & 159,831 & 24,021 & 2025 & 652,032 & 101,476 & 6.22 \\
\hline R4 & $1,238,967,329$ & -26.2 & 81 & 13 & 159,809 & 126,176 & 2025 & $1,782,523$ & 101,476 & 30.59 \\
\hline R5 & $1,232,841,636$ & -26.6 & 81 & 12 & 159,939 & 9438 & 2025 & 371,648 & 101,476 & 2.62 \\
\hline
\end{tabular}


Table 4

Capacities and costs of each platform type considered in the case study 2 .

\begin{tabular}{lll}
\hline Platform type & Capacity (bbl/d) & Cost (U.S.S) \\
\hline P1 & 60,000 & $300,000,000$ \\
P2 & 250,000 & $800,000,000$
\end{tabular}

development cost compared to the best result obtained by Rosa's methodology; the node position for the platform's installation; the quantity of horizontal wells; the total oilfield flow rate $\left(Q_{t}\right)$; the number of branch-and-cut nodes, constraints and iterations; the number of variables; and CPLEX's running time, in minutes.

According to the results, the randomness of some input data does not influence substantially on the robustness of the model. Some variability concerning the abovementioned parameters was observed, but nothing unexpected or abnormal. Optimal solutions within reasonable computational time were obtained in all cases.

A schematic drawing of the itineraries of flowlines and wells, regarding instance R5, was obtained and is shown in Fig. 2. Graphs to each level, as well as the platform installed in the node 81 and riser, are also represented.

Comparing our best case scenario (R5) with the result achieved by Rosa (2006), a significant decrease in the development cost can be highlighted, which, in the best solution of the mentioned article, was U.S.\$ $1,679,533,912$. A reduction of approximately $26.6 \%$ in the total costs, with a difference of U./S.\$ 446,692,276, was observed. Rosa's model indicated the node 80 as the best position for the platform installation. The author also adopted the previous premise that all wells are horizontal, a fact that probably contributed to the higher cost of his model.

Another improvement that can be mentioned concerns the computational time, since our experiments took, in all cases, at most a few minutes to run, while Rosa (2006) spent $16 \mathrm{~h}$ performing an exhaustive search on an Intel Pentium 4 computer of $2.4 \mathrm{~Hz}, 512 \mathrm{MB}$ of RAM and $80 \mathrm{~GB}$ hard drive. Of course it is not ideal to compare directly the performance of such distinct machines, but the use of our model clearly represents considerable savings in terms of CPU time.

\subsection{Case study 2}

This case study was based on the data of the Environmental Impact Study of Jubarte field (PETROBRAS, 2004). The Jubarte field is located in the northern portion of the Campos Basin, $77 \mathrm{~km}$ from Pontal de Ubu, south coast of Espírito Santo State, in water depths ranging between 1240 and $1350 \mathrm{~m}$.

The field has an area of $132.5 \mathrm{~km}^{2}$, which, in this work, was approximated to $144 \mathrm{~km}^{2}$, in order to facilitate the division of the field on 144 vertices $(12 \times 12)$ equally spaced with $1000 \mathrm{~m}$. The coordinates of the coast were considered to be $(77,000,300,20) \mathrm{m}$.

Originally, the oilfield development was divided into two

Table 5

Input data of case study 2 .

\begin{tabular}{ll}
\hline Case study 2: input data & \\
\hline Graph's dimensions (km) & $12 \times 12$ \\
Edge length (m) & 1000 \\
Water depth range (m) & $1240-1350$ \\
Reservoir depth range (m) & $4100-4300$ \\
Number of targets & 27 \\
Flow rate per well (bbl/d) & $3000-20,000$ \\
Maximum oil field production rate (bbl/d) & 250,000 \\
Coast coordinates X, y, z (m) & $(77,000 ; 300 ; 20)$ \\
Platform costs (U.S.\$) & 300 and 800 million \\
Platform capacities (bbl/d) & 60,000 and 250,000 \\
\hline
\end{tabular}

phases where the first one consisted in drilling four horizontal production wells connected to a production unit FPSO, called P-34, with a maximum processing capacity of 60,000 barrels of fluid per day (PETROBRAS, 2004).

For phase two, the plan forecasts the drilling of 21 production wells and 6 injection wells, all horizontal, with horizontal stretches with $1000 \mathrm{~m}$ and vertical depths between 4100 and $4300 \mathrm{~m}$. The development plan also predicts the installation of an UEP with processing capacity not defined in the document.

Given this information, we considered that there are 27 targets to be achieved by drilling, in order to also account the drilling costs of injection wells in the overall cost. The increase in the global oil flow that this consideration will result (injection wells considered like production wells) do not distance the model from reality, because, in fact, injection wells increase the oil flow of production wells. The geographical positions of the targets were randomly generated because of scarcity of data relating to these coordinates. This kind of data is provided by the Reservoir Engineering team, as a result of the steps of prospecting and exploration, and its unavailability does not invalidate the approach proposed in this work, after all, the targets chosen are distributed throughout the area of the reservoir in order to reach a maximum oil recovery.

Regarding the processing capacity of the possible UEP's, as the plan does not specify such values for phase two, we define that there are two possible sizes of platforms, with capacities and costs expressed in Table 4. The first one has the same capacity of FPSO P-34.

According to reservoir simulation, the maximum oil production expected for phase two is up to $250,000 \mathrm{bbl} / \mathrm{d}$ (PETROBRAS, 2004). That is why we chose this last value as maximum estimated oil production rate that Jubarte field can provide. Furthermore, daily oil production indicators led us to consider the flow rates ranging between 3000 and 20,000 bbl/d. The input data used on the computational experiments of this case study are briefed in Table 5 . We used the same unitary costs shown in Table 1.

The computational experiments were performed on the same software and computer of the previous case study and a similar procedure was adopted. As can be seen in Table 6, all results of the five experiments indicated that the optimum position for the platform is at node 66 and just one platform of type P2 should be installed. The lowest development cost was achieved with scenario $\mathrm{J} 3$ and, in all cases, the total oilfield flow rate is practically the maximum value, which indicates that the layout proposed by the MPWLP model utilizes the production capacity of the field in its fullness. A schematic drawing of the Jubarte field layout, concerning the solution of instance $\mathrm{J} 3$, is presented in Fig. 3 .

The actual costs expended in the Jubarte field were not published by the company. For this reason, there is no way to compare the result of the MPWLP model with the one that was applied for real. However, the values obtained appear credible for a field of this magnitude.

\section{Conclusions}

This article addressed the problem of optimizing offshore oilfield development plans by determining the number and location of production platforms, wells and manifolds; the capacities of production platforms; interconnection between platforms, manifolds and wells; and which sections of each well should be vertical or horizontal. This problem is a central element to consider in planning the development of a new field and depends on a complex association of petrophysical, geological, fluid flow behavior and operational and economic parameters. In this research we developed a novel model called Multicapacitated Platforms and 
Table 6

Results obtained for five different instances for case study 2 .

\begin{tabular}{|c|c|c|c|c|c|c|c|c|c|c|}
\hline Inst & Cost (U.S.S) & Plat. position & Plat. size & Horiz. wells & $Q_{t}(b b l / d)$ & Nodes & Const. & Iter. & Var. & Time (min) \\
\hline J1 & $1,327,863,602$ & 66 & P2 & 5 & 249,611 & 6784 & 1296 & 582,502 & 41,761 & 2.26 \\
\hline $\mathrm{J} 2$ & $1,339,702,792$ & 66 & $\mathrm{P} 2$ & 5 & 249,699 & 1068 & 1296 & 143,993 & 41,761 & 0.29 \\
\hline $\mathrm{J} 3$ & $1,314,956,409$ & 66 & P2 & 5 & 249,905 & 116,783 & 1296 & $3,052,523$ & 41,761 & 25.08 \\
\hline $\mathrm{J} 4$ & $1,351,225,856$ & 66 & $\mathrm{P} 2$ & 8 & 249,701 & 15,356 & 1296 & 419,906 & 41,761 & 1.73 \\
\hline J5 & $1,338,525,629$ & 66 & P2 & 7 & 248,948 & 5007 & 1296 & 205,180 & 41,761 & 0.51 \\
\hline
\end{tabular}

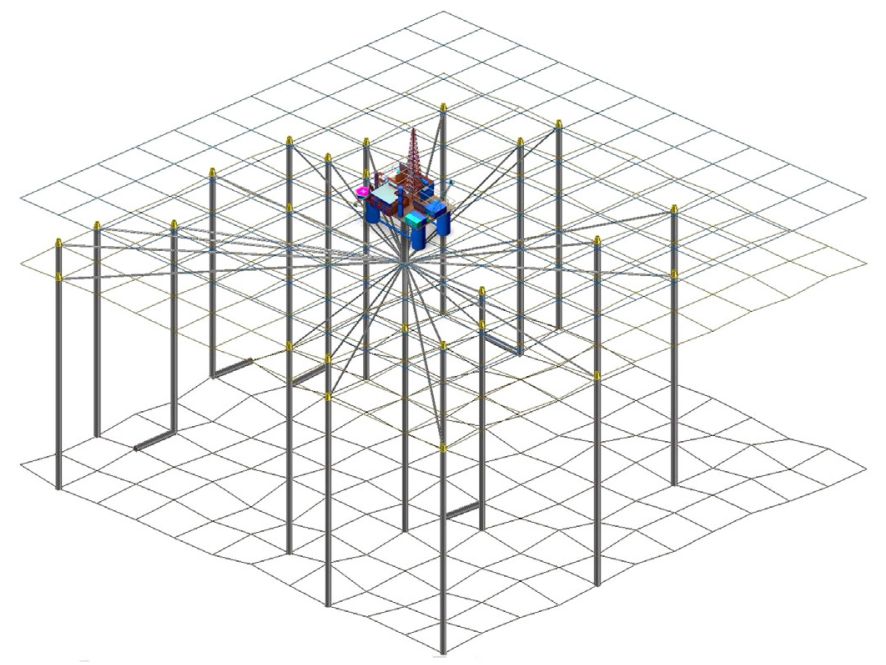

Fig. 3. Oilfield layout obtained in the computational experiment $\mathrm{J} 3$ of case study 2.

Wells Location Problem - MPWLP. The major contributions of this work and areas for further study are summarized in this chapter.

The wells and oil production platforms location problems are complex, involve many variables and combine different areas of knowledge such as Petroleum Engineering and Operational Research. Solving both problems in a single model becomes even more complicated. Additionally, there is no way to represent faithfully the actual conditions of the system and also predict future economic conditions, costs of services and facilities, taxes, market, among other factors of stochastic nature. Therefore, it was necessary to adopt several assumptions, but we strongly believe that they did not invalidate the consistency of the model, which gave us reasonable and solid results.

The idealized methodology of this work presented improvements when compared with the approaches found in literature, among which we highlight: (i) integration of two classic problems of the oil industry that are clearly interdependent, the problem of locating platforms and wells; (ii) consideration of multicapacitated platforms; (iii) inclusion of variations in depth at all levels, making the model closer to reality; (iv) consideration of the relative distance to the coast, a factor that has influence in the overall cost; (v) the model, besides deciding the positions of platforms, manifolds and wells and the platforms capacities, also indicates the best configuration for the wells (whether vertical or horizontal); (vi) just information about the location of the drilling targets in the reservoir, some unitary costs and the oil rates were necessary as input data, which let the model implementation relatively easy; (vii) the proposed approach allowed us to obtain optimal solutions, with viable computational time, for instances based on real data. The use of heuristic techniques, that do not guarantee the optimality of the obtained solution, was not required.

The solutions provided by MPWLP model can be used as a tool for decision support in conjunction with other resources to assist managers and planners in designing oilfield development plans. Moreover, it is not always true that the most economically viable alternative is also the best one when technical, safety, environmental and political aspects are considered.

The following ideas can be suggested as future enhancements for this work: (i) the application of the MPWLP proposed model in different scenarios in order to identify its limitations; (ii) integration with a reservoir simulation study to provide fluid flow data for each well candidate vertex; (iii) inclusion of the cost of manifolds installation in the objective function; (iv) an approach to maximize the NPV instead of minimizing the development costs, because this change would not only minimize costs but it would also maximize the production, which is closely related to the ducts length; (v) consideration in the variation of well flow rates with the type of well (if vertical or horizontal); (vi) consideration of directional wells; (vii) possibility of more manifolds in other places, not just immediately below its respective platforms; (viii) a multi-objective approach to the problem.

\section{Acknowledgments}

We would like to thank FUNCAP (Foundation to Support Scientific and Technological Development of Ceará) for financially support the first author during the development of this research.

\section{References}

Agência Nacional do Petróleo, Gás e Biocombustíveis - ANP 2013. Concession Contract for Exploration, Development and Production of Oil and Natural Gas (In Portuguese), p. 31.

Almeida, L.F., 2003. Selection of Alternatives for the Development of Oil Fields Using Evolutionary Computation (Master's thesis). DEEOUC/RJ, Rio de Janeiro (In Portuguese)

Bittencourt, A.C., 1997. Optimizing Hydrocarbon Field Development Using a Genetic Algorithm Based Approach. Stanford University, California, p. 1997.

Devine, M.D. Lesso, W.G. 1972. Models for the minimum cost development of offshore oil fields. Manag. Sci. 18 (8), B378-B387.

Emerick, A.A., Silva, E., Messer, B., Almeida, L.F., Szwarcman, D., Pacheco, M.A.C. Vellasco, M.M.B.R., 2009. Well placement optimization using a genetic algorithm with nonlinear constrains. In: Proceedings of SPE Reservoir Simulation Symposium. The Woodlands. SPE 118808.

Ebadat, A., Karimaghaee, P., 2012. Well placement optimization according to field production curve using gradient-based control methods through dynamic modeling. J. Pet. Sci. Eng. 100, 178-188.

Frair, L., Devine, M., 1975. Economic optimization of offshore petroleum development. Manag. Sci. 21 (12), 1370-1380.

Franco, K.P.M., 2003. Development of an Intelligent System to Assist the Choice of Offshore Production System. State University Of Campinas, Campinas, SP, p. 2003 (In Portuguese)

Grimmett, T.T., Startzman, R.A., 1988. Optimization of offshore field development to minimize investment. SPE Drilling Enginerring Meeting, 16297, http://dx.doi. org/10.2118/16297-PA. San Francisco, California, USA.

Guyaguler, B., Horne, R., 1999. Optimization of well placement. Journal of Energy Resources Technology 122 (2), 64-70. http://dx.doi.org/10.1115/1.483164.

Hansen, P., Pedrosa Filho, E.L., Ribeiro, C.C., 1992. Location and sizing of offshore production for oil exploration. Eur. J. Oper. Res. 52, 202-214.

Pacheco, M.A.C., Vellasco, M.M.B.R., 2009. Intelligent systems in oil field development under uncertainty. Studies in Computational Intelligence. Springer Berlin/ Heidelberg, p. 288

Petrobras - Petróleo Brasileiro S.A., 2004. Environmental Impact Study: Production Activity and Flow Oil and Gas of Jubarte field in the Campos Basin - Technical Report (In Portuguese). Vitória, p. $1-88$.

Rahmawati, S.D., Whitson, C.H., Foss, B., Kuntadi, A., 2012. Integrated field operation and optimization. J. Pet. Sci. Eng. 81, 161-170 (Elsevier). 
Rosa, V.R. Platform Location Optimization (In Portuguese), 2006. 116 f. Master's thesis (Master of Science in Production Engineering) - Alberto Luiz Coimbra Post Graduate and Engineering Research Institute (COPEE), Federal University of Rio de Janeiro, Rio de Janeiro. 2006.

Rosa, V.R.; Ferreira Filho, V.J.M. Optimizing the location of platforms and manifolds In: International Conference on Ocean, Offshore and Arctic Engineering - OMAE 2012, Rio DE Janeiro. Proceedings of the ASME 2012 31st International Conference on Ocean, Offshore and Arctic Engine. pp. 1-6. 2012.

Sarmiento, A.M., Nagi, R., 1999. A review of integrated analysis of production- distribution systems. IIE Trans. 31, 1061-1074.

Tavallali, M.S., Karimi, I.A., Teo, K.M., Baxendale, D., Ayatollahi, S., 2013. Optimal producer well placement and production planning in an oil reservoir. Comput. Chem. Eng. 55, 109-125 (Elsevier).

Túpac, Y.J., 2005. Intelligent System for Optimization of Alternatives for the Development of Oil Fields (Ph.D. thesis). DEE - PUC/RJ, Rio de Janeiro (In Portuguese).

Yeten, B., 2003. Optimum Deployment of Nonconventional Wells (Ph.D. thesis). Stanford University, California, p. 2003. 